K ul t s chi tz ky : Beitr. z. Frage üb. d. Verbreitung d. glatten Muskulatur etc. 15

\title{
Beitrag zur Frage über die Verbreitung der glatten Muskulatur in der Dünndarmschleimhaut.
}

\author{
Von
}

Dr. med. Kultsehitzky,

Prosector und Privat-Docent der Histologie an der Universität zu Charkoff.

Hierzu Tafel II.

Die unzweifelhafte Anwesenheit contractiler Elemente in den Zotten war von Prof. Brücke ${ }^{1}$ ) (1851) erwiesen, als es diesem Autor gelang, die einzelnen Muskelzellen zu isoliren. Brticke glaubte, dass die muskulïren Elemente sich in Form einzelner Bundel von der Basis der Zotte parallel der Axe der letzteren bis zur Spitze erstrecken und dort enden. Diese Art der Verbreitung der Muskeln wurde, wie es scheint, von den meisten Autoren angenommen (Kölliker ${ }^{2}$ ), Dönitz ${ }^{3}$ ) u. a.).

Basch nimmt ebenfalls an, dass in der Zotte einzelne Muskelbündel verlaufen, die parallel zur Längsaxe dersclben gelegen sind. Zur centralen Höhle sollen dieselben nach Basch concentrisch gelegen sein. Die am meisten nach innen gelegenen Bündel, welche zugleich die stärksten sind, umgrenzen unmittelbar die centrale Höhle. Die äusseren, zugleich die schwächeren, verlaufen mitten im Parenchym der Zotte und enden frei an ihrer Spitze ${ }^{4}$ ).

Ein ganz anderes Verbalten bescbrieb Verson ${ }^{5}$ ). Nach

1) Brücke, Ueber ein in der Darmschleimhaut anfgefundenes Muskelsystem. Sitzungsber. der mathem.-naturw. Classe der Wiener Akademie Bd. VI, p. 214.

2) Kölliker, Gewebelehre.

3) Dönitz, Arch. für Anatomie, Physiologie und wiss. Medicin 1864, p. 404 .

4) B asch, Das Zottenparenchym und die ersten Chyluswege. Sitzungsb. der Wiener Akad. 1865, Bd. LI, p. 5.

5) Verson, Stricker's Handbuch der Gewebelehre 1877, Bd. I, p. 408. 
seinen Untersuchungen bilden die Muskelfasern ron der Muscularis mucosae ausgehend Schlingen $\mathfrak{u m}$ das blinde Ende der Zottenhöhle und kehren dann zur Muscularis mucosae wieder zurïck. Ein solches Verhalten beobachtete Verson bei fast ausgetragenen Meerschweinchenembryonen und bei erwachsenen Katzen.

v. Thanhoffer ${ }^{1}$ ) nimmt in den Dinndarmzutten ausser den parallel der Längsaxe verlaufenden glatten Muskelfaserbündeln (in Uebereinstimmung mit Basch) noch die Anwesenheit von quer oder circulär verlaufenden muskulären Elementen an. Und zwar stiutzt dieser Autor seine Annahme darauf, dass er auf den Längsschnitten der Zotten quergelegene stäbchenförmige Kerne beobachtete, die bald an der Spitze, bald an der Mitte, bald an der Basis der Zotte lagen. Die Grösse der Kerne sowohl quer als längs verlaufender Muskelzellen sind nach v. Thanhoffer ganz gleich. Ausser den erwähnten quer in den oberflächlichsten Schichten verlaufenden muskulären Elementen können solebe nach $v$. Than hoffer auch in den tieferen Schichten der Zotte (wenigstens bei Hunden) nachgewiesen werden.

Noch vor $\nabla$. Than h offer wurden querverlaufende Muskelelemente in den Zotten von Molesch ott besehrieben.

Fortunato $f^{2}$ ) beobachtete in den Hundezotten (in Uebereinstimmung mit $\nabla$. Thanhoffer) oberflächlich gelegene querverlaufende muskuläre Elemente und tiefe längsverlaufende. Ansserdem fand er querverlaufende Muskelzellen auch in der Mitte der Zottenmasse, schwankt aber in dem Schlusse, ob dieselben den längs- oder querverlaufenden Bündeln angehören, da die längsverlaufenden oft Anastomosen bilden, welche auf dem Querschnitte für querverlaufende gehalten werden können.

Aus den Abhandlungen der letzten Zeit treffen wir ausfïhrlichere und genauere Angaben über die Verbreitung der Muskeln in der Dünndarmzotte in der Abhandlung von F. Graf Spee ${ }^{3}$ ). Wir wollen hier

1) v. Than hoffer, Beiträge zur Fettresorption und histologischen Structur der Dünndarmzotten. Arch. f. die gesammte Physiologie Bd. ViI, 1873.

2) Fortunatoff, Die Fettresorption und histologische Structur der Dünndarmzotten. Arch. f. die gesammte Physiol. Bd. XIV, 1877, p. 289.

3) F. Graf Spee, Beobachtungen über den Bewegungsapparat und die Bewegung der Darmzotten, sowie deren Bedeutung für den Chylusstrom. Arch. f. Anatomie und Physiologie. Anatomische Abtheilung 1885, p. 160-188. 
Beitrag zur Frage über die Verbreitung der glatten Muskulatur etc. 17

nur die dort vorbandenen Angaben tiber den Verlauf der Muskelfasern in den Zotten des Hundes wiedergeben, da wir den Darm des letzteren als Object für unsere Beobachtungen benutzt haben.

Nach Spee verlaufen die Muskelbtindel in der Zotte entweder ganz parallel der Längsachse der letzteren oder, was aber nur selten vorkommt, etwas von dieser Richtung abweichend. Die Hauptmuskelbïndel liegen dem Centralcanal an, ein Theil derselben liegen in einiger Entfernung im Parenchym der Zotte. Die Muskelbündel enden oben an. der Spitze der Zotte, wobei sie entweder bogenförmig zusammenlaufen oder Schlingen um die Blutgefässe bilden können. Sie können auch zuweilen an den seitlichen Theilen der Zotte bis zum Epithelium reichen, ?dasselbe kommt aber nur als Ausnahme vor.

Spee sagt unter anderem, dass er bei seinen Untersuchungen tiberhaupt den Eindruck erhalten habe, dass die Muskelbündel an der Basis der. Zotte etwas dichter um den Centralcanal aneinander gelagert sind, um ihm bis zur Muscularis mucosae zu folgen, wobei siein derSchicht der L i eber $\mathrm{k}$ ü $\mathrm{h} \mathrm{n}$ 'schen Drlisen an Mächtigkeit abnehmen ${ }^{1}$ ). Querverlaufende Muskelbundel konnte S pee niemals finden.

Die Schlussfolgerung Spee's, die wenig von derjenigen der anderen Autoren abweicht, ist folgende: Es kann angenommen werden, dass bei allen Thieren die Muskelbündel nur längs der Zotte verlaufen und der Endothelwand des Centralcanals anliegen.

Nach diesem kurzen literarischen Abriss gehe ich zur Beschreibung meiner eigenen Untersuchungen über diesen Gegenstand über. Zuvor möchte ich aber noch einige Bemerkungen iiber die von mir gebrauchten Untersuchungsmethoden vorausschicken.

Zur Fixirung des Hundedarmes, der mir, wie bereits erwähnt, als aussebliessliches Untersuchungsobject diente, benutzte ich eine Mischung von folgender Zusammensetzung :

a) gesättigte Lösung von Kali bichromicum und Cuprum sulfuricum in $50^{\circ}$ Alkohol (im Dunkeln) und

b) eine kleine Quantität Essigsäure (ungefähr 5-6 Tropfen an $100 \mathrm{ccm}$ ), welche unmittelhar vor dem Fixiren hinzugefügt werden muss.

1) 1. c. p. 171.

Archiv f, mikroak. Anatomie. Bd. 31. 
In dieser Mischung blieben die Objecte 24 Stunden, wurden dann in Alkohol absolutus übertragen und hernach in Paraffin eingebettet. Sowohl das Fixiren, als das Erhärten muss im Dunkeln vor sich gehen, um das Absetzen der gebrauchten Salze zu verhindern.

Zum Färben benutzte ich eine unlängst von mir veröffentlichte sauere Chloralhydratcarminlösung ${ }^{1}$ ), welche gerade hier sehr gute Dienste leistet, da diese Lösung nicht nur die Kerne selbst fürbt, sondern auch die Maskelzellenkörper und zwar letztere etwas anders als die äbrigen Theile des Präparates.

Vermöge dieser Färbung treten die Muskeln besonders deutlich hervor und können bis zu ihren Ansatzpunkten verfolgt werden.

Bei meinen Untersuchungen benutzte ich das Jung'sche Microtom und die Zeiss'schen Objectivlinsen 1/12 Oelimmers. und Oel-Apochromat (Brennw. 2,0, n. A. 1,40).

Wir wollen unsere Beobachtung des Verlaufes der Muskelbïndel in der Dünndarmschleimhaut mit dem Orte ihres Anfanges beginnen, d. h. mit der Muscularis mucosae. Letztere besteht beim Hunde wie bekannt aus 2 resp. aus 3 Schichten. Wie es scheint werden für die Sehleimhaut bestimmte Muskelbündel von allen Schichten der Muscularis mucosae geliefert. Diese Bündel gehen zunächst schräg nach oben, wobei sie zu den Lieberkühn'schen Drisen durch eine ziemlich dicke, flach unter derselben gelegene Schicht des Grundgewebes gelangen müssen.

An diesen Drüsen angelangt, laufen die Muskelbündel parallel der Längsaxe derselben weiter fort. Nach meinen Untersuchungen stehen die Muskelbtindel in der Schicht der Lieberkühn'schen Drüsen in keiner bestimmten Beziehung zu den Chylusgefässen dieser Schicht, wie ich den Angaben Graf Spee's gegentiber behaupten muss. An der Basis der Zotte angelangt, häufen sich dieselben dichter aneinander an und legen sich zugleich an das aus der Zotte gehende Chylusgefäss resp. an den sogenannten Centralcanal der Zotte an.

1) Kultschizky, Zur histologischen Technik. Zeitschrift f. wissensch. Mikroskopie Bd. IV, 1887, p. $46-49$. 
An der Basis der Zotte und in einiger Entfernung nach dem Innern derselben sind die Muskelbündel am dichtesten aneinander gelagert, was übrigens auch mit den Angaben von Spee tubereinstimmen wïrde. Wir müssen aber betonen, dass die Muskeln im Bereiche der Lieberkühn'schen Drüsen keineswegs an Mächtigkeit abnehmen, wie es Spee angibt, sondern dieses scheinbare Schwächerwerden derselben rübrt von der Art ihrer Vertheilung her. Indem nämlich die Bündel im Bereiche der Lieber $\mathrm{k} u ̈ h \mathrm{~h}$ 'schen Drüsen dünn und zerstreut verlaufen, treten dieselben hier nicht so plägnant hervor, dagegen legen sich die Bündel an der Basis der Zotte dichter aneinander an und bilden eine fast ununterbrochene Muskelschicht um das Chylusgefäss herum und erscheinen deshalb die Muskelbündel hier viel grösser als dort.

Diese Thatsache ist von mir in einer frtiheren Abhandlung über diesen Gegenstand bereits hervorgehoben worden ${ }^{1}$ ).

Nach Basch und Spee liegen, wie oben bereits angegeben ist, die Muskelbündel in den Zotten des Hundes dem Centralcanal an (Hauptmasse) und ausserdem (ein verbältnissmässig kleiner Theil der ganzen Muskulatur der Zotte) einzelne Bündel im Stroma der Zotte, wobei die Hauptbündel parallel der Längsaxe verlaufen. Diese Angabe kann ich durchaus bestätigen und am die Wiederholung bereits bekannter Thatsachen zu vermeiden, werde ich mich dabei nicht länger aufhalten. Ich will nur hinzufügen, dass es zwischen den einzelnen Bündeln eine grosse Anzahl Anastomosen gibt, die sebr oft die Dicke der Hauptbündel erreichen.

Dem gegenüber existirt in unseren Kenntnissen über die Muskeln der Zotten eine grosse Lücke und diese muss zuerst ausgefullt werden, wenn wir den Mechanismus der Zottenmuskulatur verstehen sollen. Sämmtliche Autoren, auch $\mathrm{S}$ pee, dessen Abhandlung als die genaueste bezeichnet werden muss, nicht ausgenommen, liessen einen Punkt fast ganz unberïcksichtigt, jedenfalls unentschieden, nämlich die Frage über die Ansatzpunkte der Zottenmuskeln. Wir kennen einen der Ansatzpunkte der Muskelbündel, wir wissen, dass dieselben in der Muscularis mucosae ihren Anfang nehmen. Dieses genügt aber nicht, um die physiologische

1) Zur Frage nach dem Bau der Düundarmschleimhaut und dem Mechanismus der Resorption. Charkoff 1882 (Russisch). Refer. im Jahresber. von Hofmann und Schwalbe, Literatur 1883, p. 236-237. 
Kultschitzky:

Wirkung eines Muskels zu beurtheilen, dazu müssen wir noch den zweiten Ansatzpunkt desselben kennen. In Anbetracht der Wichtigkeit dieser Frage richtete ich bei meinen Untersuchungen mein Hauptaugenmerk auf diesen Gegenstand und gelangte $\mathrm{zu}$ folgendem Ergebnisse.

Von der Basis der Zotte beginnend gehen die Muskelbündel nach oben parallel der Laingsachse derselben, geben aber während ihres ganzen Verlaufes Zweige, die schief nach oben und zur Peripherie der Zotte geben, ab. Diese Zweige erreichen das Epithel und setzen sich sofort unter demselben an. Die ebenerwähnten Zweige allmählich abgebend werden die Bündel immer dünner, ein Theil derselben reicht aber bis zur Spitze der Zotte hin. Dort gehen die Bündel pinselförmig auseinander, erreichen das Epithel und setzen sich ebenfalls gleich den erwähnten Seitenzweigen dicht unter demselben an; es war mir aber bisher unmöglich zu entscheiden, in welchen Gewebselementen die feinen Muskelausläufer eigentlich ihr Ende finden.

Diesen meinen Beobachtungen zufolge setzt sich die ganze Masse der in die Zotte eingetretenen Muskelbündel an der ganzen Innenfläche (unmittelbar anterdem Epithel) derselben an. Die beigegebenen Abbildungen sind verschiedenen Gebieten entnommen. Fig. I aus dem Scheiteltheil, Fig. II aus dem Mitteltheil und Fig. III nahe der Basis. Dieser mikroskopische Befund berechtigt uns za einigen Schlussfolgerungen tiber die physiologische Rolle der Zottenmuskulatur.

Was diejenigen Bündel anbelangt, die parallel der Axe der Zotte verlaufen, so ist ihre Wirkung leicht verständlich: sie bewirken, wie es beteits $\mathrm{Br}$ iu ck e ausgesprochen hat, wälrend ihrer Zusammenziehung die Verkürzung der Zotte. Dagegen haben diejenigen Bündel, welche schief nach oben gehen und an der Peripherie sich ansetzen, eine etwas andere Bestimmung, ob zwar sie ebenfalls die Verkürzung der Zotte bewirken. Wir haben frtiher gesehen, dass die Muskelbiindel während ibres Verlaufes zwischen den Lieberkithn'schen Drïsen und der Zottenbasis eine convergirende Richtung innehalten, dass sie sich dann an das Chylusgefäss anlegen und allmählich auseinanderweichen. Demnach haben die Muskelbündel die Form von Bögen, deren convexe Seiten der Chylusgefässwand anliegen. Die Muskelbündel streben selbstrerständlich bei ihrer Contraction eine geradlinige 
Beitrag zur Frage über die Verbreitung der glatten Muskulatur etc. 21

Richtung einzunehmen; da aber die Punkte, an welchen die convexen Stellen der Bündel anliegen (die Chylusgefässwand), leicht beweglich sind, so werden dieselben im Momente der Muskelcontraction nach allen seiten ausgezogen werden und zwar senkrecht zur Längsaxe der Zotte, oder mit anderen Worten, der Centralcanal der Zotte wird sich erweitern. Somit können wir durch Berücksichtigung der Art des Verlaufes der Muskelbündel eine Thatsache erklïren, die bereits von Spee constatirt worden war, nämlich, dass in der contrahirten Zotte das Chylusgefäss breiter ist als in der ruhenden ${ }^{1}$ ).

Als logische Schlussfolgerung aus der eben angedeuteten Rolle der Muskelbiindel (Erweiterung des Centralcanals der Zotte) kann folgender Satz ausgesprochen werlen: Der Centraleanal bleibt, dank der auseinandergesetzen Muskelverthei lung, während der ganzen Contractionszeit der Zotte offen erhalten, mit anderen Worten, während dieses ganzen Zeitraumes ist der freie Abflussdes Milebsaftes vollständig gesichert.

Zum Schlusse möchte ich noch mit einigen Worten der von v. Thanhof fer besonders betonten Thatsache Erwägung thun, nämlich des Vorhandenseins von querverlaufenden Muskelfasern in den Zotten. Bei meinen Untersuchungen Labe ich mich in Uebereinstimmung mit vielen anderen Autoren iberzeugen können, dass in den Zotten keine derartigen querverlaufenden Fasern existiren. Auf dünnen, aus einer Hundedarmzotte verfertigten Schnitten sind leicht längliche, quer zur Zottenlängsaxe gerichtete Kerne zu finden, welche sehr den Kernen der glatten Muskelfasern ähnlich sehen und sind sogar kaum von Muskelfaserkernen $\mathrm{zu}$ unterscheiden. Dieser Befund gab offenbar Veranlassung von querverlaufenden Muskelfasern zu sprechen, indess lässt sich bei genauerer Untersuchung (mit Hülfe des Apochromats) feststellen, dass diese Kerne nicht Muskeln, sondern ausgezogenen Zellen des Zottenbindegewebes angehören.

1) 1. c. p. 183 . 


\section{Erklärung der Abbildnngen auf Tafel II.}

Sämmtliche Abbildungen sind mittelst des $\mathrm{A}$ b b é'schen Zeichenapparates und des Zeiss'schen Apochromates (2,0 Brennw. n. A: 1,40) hergestellt.

Fig. 1 ist nachträglich auf die Hälfte reducirt und stellt den Verlauf und den Ansatz der Muskelbündel an der Spitze der Zotte dar.

Fig. 2. Ansatz der Muskelbündel im mittleren Theile.

Fig. 3. Dasselbe an der Basis der Zotte.

(Aus dem anatomischen Institute des Prof. Dr. E. Zuckerkandl in Graz.)

\section{Ueber das Vorkommen von Nerven-Endkörperchen in der männlichen Harnröhre.}

Von

\section{Dr. Richard v. Planner,}

em. Assistent an der dermatol. Lebrkanzel in Graz.

Hierzu Tafel III.

Wer sich mit Erkrankungen des Urogenitalsystems beschäftigt, macht die Erfahrung, dass die nervösen Störungen innerhalb desselben beztiglich der Häufigkeit nächst den einfach catarrbalischen den ersten Platz einnehmen. Ja, man kann sagen, dass nahezu jede Erkrankung, bei der es zu ausgesprochenen anatomischen Veränderungen der Schleimbaut des Urogenitalsystems kommt, von mehr oder weniger deutlichen nervösen Alterationen gefolgt ist. Während dies für das weibliche Geschlecht in Folge der gewöhnlich ganz eclatanten Erscheinungen schon lange hinlänglich gewürdigt ist, wurde beim männlichen Geschlechte derartigen Erkrankungen erst in jüngster Zeit gebührende Aufmerksamkeit geschenkt und verdanken wir insbesondere den Ausführungen U1tz- 